\title{
Simultaneous Left Ventricular Pseudo-Aneurysm and Ventricular Septal Rupture After a Non-ST Segment Myocardial Infarction
}

\author{
Carlos D. Davila ${ }^{\mathrm{a}, \mathrm{d}}$, Leandro Slipczuk ${ }^{\mathrm{b}}$, Shushita Gupta ${ }^{\mathrm{c}}$
}

\begin{abstract}
The incidence of myocardial rupture following an acute coronary syndrome has dramatically decreased with the implementation of early reperfusion strategies. High clinical suspicion is critical for early recognition and appropriate management of myocardial mechanical complications. Here we present an uncommon combination of left ventricular pseudo-aneurysm and ventricular septal rupture complicated with coronary sinus shunting following a non-ST elevation myocardial infarction, where modern echocardiography allowed prompt and accurate identification of the event.
\end{abstract}

Keywords: Myocardial rupture; Pseudo-aneurysm; Echocardiography

\section{Introduction}

Morbidity and mortality associated with acute myocardial infarction (AMI) have steadily decreased in the post re-perfusion era, nevertheless cardiac rupture occurs in about $4 \%$ of patients after AMI [1]. The entire spectrum of cardiac rupture was responsible for $15 \%$ of early death in the Thrombolysis in Myocardial Infarction Phase II study (TIMI II) [2].

Ventricular septal rupture (VSR) formerly occurred in $1-2 \%$ of patients after AMI but the incidence has dramatically decreased [3]. In a small number of cases, dilatation and remodeling of the damaged ventricular wall without rupture may lead to ventricular aneurysm formation [4]. Conversely in approximately $0.2-0.3 \%$ of infarctions, complete cardiac rupture

Manuscript accepted for publication December 03, 2014

aDepartment of Medicine, Einstein Medical Center, Philadelphia, PA, USA

${ }^{b}$ Heart Institute, Cedars-Sinai Medical Center, Los Angeles, CA, USA

'Division of Cardiology, Einstein Medical Center, Philadelphia, PA, USA

${ }^{\mathrm{d} C}$ Corresponding Author: Carlos D. Davila, Albert Einstein Medical Center, 5501 Old York Road, Klein 363, Philadelphia, PA 19141, USA.

Email: davilacc@einstein.edu

doi: http://dx.doi.org/10.14740/jmc2005w is contained by adjacent tissue, most often the pericardium, forming a pseudo-aneurysm [5]. Differentiation between left ventricular (LV) aneurysm and pseudo-aneurysm is difficult, yet it is extremely important given the greater propensity for rupture of the latter, hence requiring emergent surgical repair $[1,6,7]$. High index of suspicion is needed to determine the appropriate therapeutic approach and prognosis in patients with these complications. Several imaging modalities are used to discriminate between different types of myocardial mechanical complications [8]. We present a case of a patient with simultaneous LV pseudo-aneurysm and VSR complicated with coronary sinus shunting secondary to a non-ST-segment elevation myocardial infarction (NSTEMI).

\section{Case Report}

A 76-year-old Jamaican female with history of hypercholesterolemia, hypertension and diabetes type II presented with congestive heart failure following an NSTEMI that had occurred 2 weeks prior to admission. Patient had undergone percutaneous revascularization to the right coronary artery with a drug eluting stent for a severe stenotic lesion and was discharged on dual antiplatelet therapy and optimal medical management to a

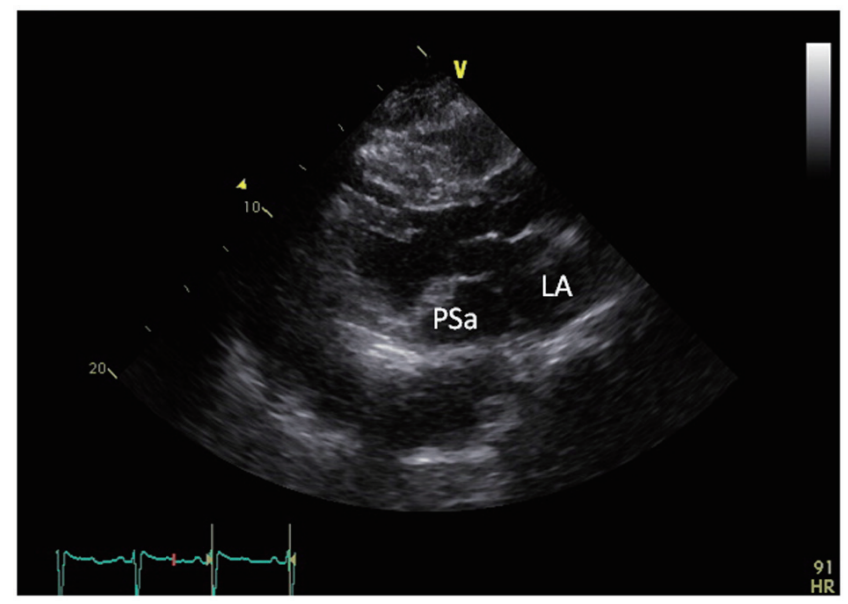

Figure 1. Transthoracic echocardiogram, parasternal long axis view showing the ruptured pseudoaneurysm compressing the left atrium. LA: left atrium; PsA: pseudo-aneurysm. 


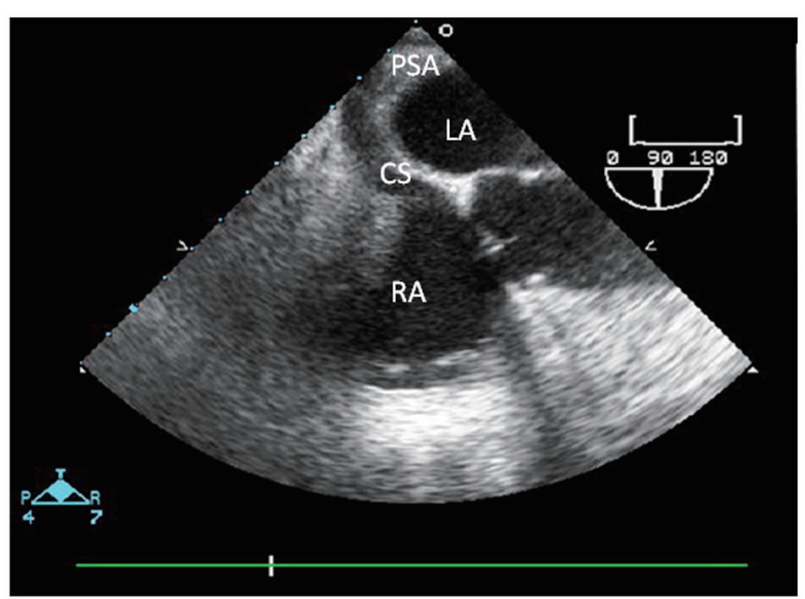

Figure 2. Transesophageal echocardiogram, modified bicaval view showing the coronary sinus and the pseudo-aneurysm. LA: left atrium; RA: right atrium; PsA: pseudo-aneurysm; CS: coronary sinus.

skilled nursing facility.

During re-admission she was in NYHA Class III heart failure, which had progressed from NYHA class I 2 weeks prior, with rapid worsening in the last 2 days. The electrocardiogram showed sinus tachycardia, left axis deviation and Q waves in inferior leads. Chest radiographs showed cardiomegaly; the hemogram and biochemical parameters were all relatively normal except for mild acute kidney injury.

Trans-thoracic echocardiogram showed an ejection fraction of $55 \%$, LV thinning and severe hypokinesis of the inferior wall with large aneurysm formation and mild to moderate mitral regurgitation. Right heart chambers were normal in size; the pulmonary artery systolic pressure was calculated to be 60 $\mathrm{mm} \mathrm{Hg}$. A trans-esophageal echocardiogram revealed an LV inferobasal aneurysm with rupture forming a pseudoaneurysm and shunting into the coronary sinus, resulting in a left to right shunt (Figure 1-3).

Patient was placed on hemodynamic support with an intraaortic balloon pump and subsequently taken to the operating room for surgical repair of the pseudoaneurysm. Intra-operatively a VSR was also noticed. She underwent LV pseudoaneurysm repair, VSR repair with a pericardial patch followed by pericardial washout and chest wall closure. She was transferred to the coronary care unit with clinical and hemodynamic improvement in the following days.

\section{Discussion}

Acute coronary syndrome (ACS) accounted directly or indirectly for $25 \%$ of all deaths in the United States in the last decade [9]. Myocardial rupture occurs in $4 \%$ of the cases as a complication from several types of injuries, more often, myocardial infarction (MI) [1]. Ventricular free wall rupture occurs in the majority of cases and is usually associated with cardiogenic shock and death secondary to cardiac tamponade [10].

Cardiac rupture may be broadly divided into: 1) complete or incomplete; 2) free ventricular wall rupture or rupture of

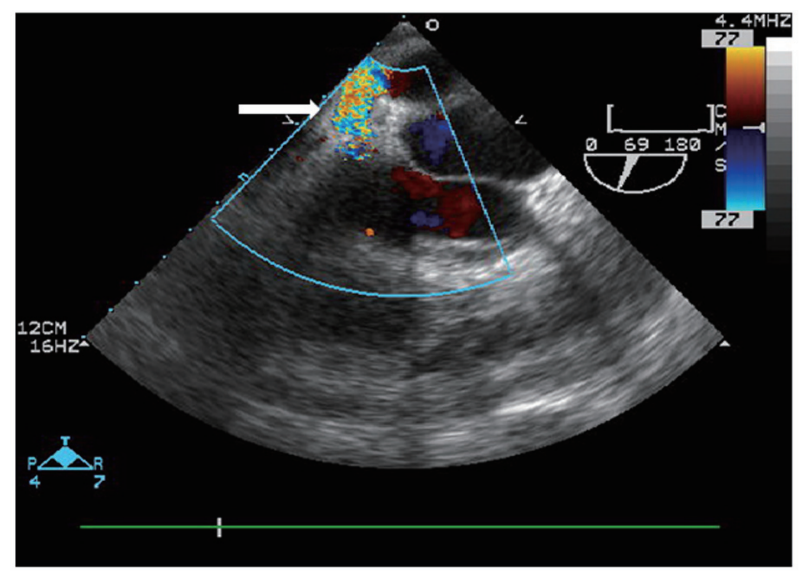

Figure 3. Transesophageal echocardiogram, modified bicaval view. Arrow shows abnormal flow from coronary sinus to pseudo-aneurysm.

intracardiac structures such as the interventricular septum and/ or the papillary muscles. The pathophysiology and mechanical determinants of myocardial rupture can be explained by wall stress and stress tolerance. Wall stress follows LaPlace's law [11], and stress tolerance relates to diseases or conditions which lower the level of injury tolerated by the myocardial muscle and surrounded structures, most commonly ischemic necrosis, but also previous scars, cardiac surgery and trauma.

The Global Registry of Acute Coronary Events (GRACE), which is a multinational registry involving 30 countries designed to reflect an unbiased population of patients across the full ACS spectrum, showed that heart rupture was more common in females, older patients, following an ST-segment elevation MI (STEMI) with higher level of cardiac enzymes than those without myocardial rupture. In addition, higher heart rate and lower blood pressure at the time of admission were independently associated with cardiac rupture. Thrombolytic treatment was used more frequently in patients who sustained rupture. Conversely, chronic ischemic heart disease, history of invasive revascularization procedures, beta-blockers and low molecular weight heparin administration were observed less commonly in patients with heart rupture. The GRACE score, originally designed to predict in-hospital death was associated with myocardial rupture only in an unadjusted model (OR $1.20,95 \%$ CI $1.16-1.23$ ) and therefore should not be used to predict the incidence of it $[12,13]$.

Ventricular pseudo-aneurysm is considered an incomplete type of myocardial rupture, given the temporal confining by a portion of the surrounding pericardium. In contrast to a true aneurysm, the walls of a pseudo-aneurysm lack the structural elements found in the normal myocardial wall. Ventricular pseudo-aneurysm is a rare phenomena occurring in less than $0.5 \%$ of myocardial infarction [14]. According to previous publications, ventricular pseudo-aneurysm are more often located in the infero-posterolateral walls (82\%) and associated with right coronary artery (RCA) and circumflex disease as culprit lesion for MI [15]. Diagnosis of pseudo-aneurysms used to rely on cardiac catheterization and contrast ventriculogram [16], now it is commonly detected with modern two-dimensional Doppler echocardiography and color flow imaging. Typically the 
maximal internal width of the aneurysmal fundus (Dmax) is larger than the maximal internal diameter of its neck or orifice (Omax) on a same frame (Gatewood and Nanda index), also with the aid of color Doppler flow, pseudo-aneurysm can be confirmed by an unusual continuous flow signal extending from the LV cavity to the aneurysmal cavity through its narrow neck [8]. However in very large pseudo-aneurysms definition of the orifice and fundus can be challenging. M-mode echocardiography does not appear to be sensitive or specific in this diagnosis [17]. Other imaging modalities like computed tomography angiography and cardiac MRI have a limited role in the diagnosis of these conditions, although they have helped to understand the process of their formation. Currently they are being used as research tools in small case series and as part of follow- up in patients with these complications [18]. As a caveat cardiac MR is still not universally available and its cost is higher than other methods.

Given the propensity of pseudo-aneurysm to rupture, surgical repair is required immediately after the diagnosis has been made. Mortality can be as high as $90 \%$ if left untreated [19].

True aneurysms are located in the anterior myocardial wall and the apex in up to $93 \%$ of cases; typically they are formed 2 - 3 weeks after an event correlated with the left anterior descendent artery (LAD). The aneurysmal sac is composed of myocardial fibers and has a wide communication between the LV cavity and the sacular deformity [20]. Eventually this area will develop hypo and akinesia with increase risk of thrombus formation and embolic events [21]. Over 95\% of aneurysms are secondary to coronary artery disease; other causes include coronary artery malformation, sarcoidosis, Chagas' disease, arrhythmogenic right ventricular dysplasia and even idiopathic causes [22]. The most important fundamental feature differentiating a true aneurysm from false aneurysm is the presence of a continuous surrounding myocardial wall by echo [23]. Ventricular aneurysm can be treated medically and if necessary with elective surgery [24].

VSR and papillary muscle rupture (PMR) are considered early mechanical complications following an MI. The GUSTO-I trial demonstrated an incidence of VSR of approximately $0.3 \%$ and PMR of $0.9 \%$, which are even lower after the introduction of primary percutaneous intervention in the setting of acute MI. Echocardiography, in combination with Doppler, serves as the preferred method for the detection and differentiation of these two entities [25].

\section{Conclusion}

Mechanical complications after AMI are rare but still prevalent. Most publications and cases focus on mechanical complications after STEMI. To the best of our knowledge, there are no ante-mortem cases in the literature with the combination we have presented $[26,27]$. We herein reported a case of simultaneous LV pseudo-aneurysm accompanied with VSR after an NSTEMI, in which modern echocardiography and high clinical suspicion prompted appropriate management and good outcomes.

\section{References}

1. Becker RC, Gore JM, Lambrew C, Weaver WD, Rubison RM, French WJ, Tiefenbrunn AJ, et al. A composite view of cardiac rupture in the United States National Registry of Myocardial Infarction. J Am Coll Cardiol. 1996;27(6):1321-1326.

2. Kleiman NS, Terrin M, Mueller H, Chaitman B, Roberts R, Knatterud GL, Solomon R, et al. Mechanisms of early death despite thrombolytic therapy: experience from the Thrombolysis in Myocardial Infarction Phase II (TIMI II) study. J Am Coll Cardiol. 1992;19(6):1129-1135.

3. Moreyra AE, Huang MS, Wilson AC, Deng Y, Cosgrove NM, Kostis JB. Trends in incidence and mortality rates of ventricular septal rupture during acute myocardial infarction. Am J Cardiol. 2010;106(8):1095-1100.

4. Gorlin R, Klein MD, Sullivan JM. Prospective correlative study of ventricular aneurysm. Mechanistic concept and clinical recognition. Am J Med. 1967;42(4):512-531.

5. Csapo K, Voith L, Szuk T, Edes I, Kereiakes DJ. Postinfarction left ventricular pseudoaneurysm. Clin Cardiol. 1997;20(10):898-903.

6. Van Tassel RA, Edwards JE. Rupture of heart complicating myocardial infarction. Analysis of 40 cases including nine examples of left ventricular false aneurysm. Chest. 1972;61(2):104-116.

7. Eren E, Bozbuga N, Toker ME, Keles C, Rabus MB, Yildirim O, Guler M, et al. Surgical treatment of postinfarction left ventricular pseudoaneurysm: a two-decade experience. Tex Heart Inst J. 2007;34(1):47-51.

8. Gatewood RP, Jr., Nanda NC. Differentiation of left ventricular pseudoaneurysm from true aneurysm with two dimensional echocardiography. Am J Cardiol. 1980;46(5):869-878.

9. Erne P, Gutzwiller F, Urban P, Maggiorini M, Keller PF, Radovanovic D. Characteristics and Outcome in Acute Coronary Syndrome Patients with and without Established Modifiable Cardiovascular Risk Factors: Insights from the Nationwide AMIS Plus Registry 1997-2010. Cardiology. 2012;121(4):228-236.

10. Dellborg M, Held P, Swedberg K, Vedin A. Rupture of the myocardium. Occurrence and risk factors. Br Heart J. 1985;54(1):11-16.

11. Zhong L, Ghista DN, Tan RS. Left ventricular wall stress compendium. Comput Methods Biomech Biomed Engin. 2012;15(10):1015-1041.

12. Rationale and design of the GRACE (Global Registry of Acute Coronary Events) Project: a multinational registry of patients hospitalized with acute coronary syndromes. Am Heart J. 2001;141(2):190-199.

13. Lopez-Sendon J, Gurfinkel EP, Lopez de Sa E, Agnelli G, Gore JM, Steg PG, Eagle KA, et al. Factors related to heart rupture in acute coronary syndromes in the Global Registry of Acute Coronary Events. Eur Heart J. 2010;31(12):1449-1456.

14. Roberts WC, Morrow AG. Pseudoaneurysm of the left ventricle. An unusual sequel of myocardial infarction and rupture of the heart. Am J Med. 1967;43(4):639-644. 
15. Mackenzie JW, Lemole GM. Pseudoaneurysm of the left ventricle. Tex Heart Inst J. 1994;21(4):296-301.

16. Higgins $\mathrm{CB}$, Lipton $\mathrm{MJ}$, Johnson $\mathrm{AD}$, Peterson $\mathrm{KL}$, Vieweg WV. False aneurysms of the left ventricle. Identification of distinctive clinical, radiographic, and angiographic features. Radiology. 1978;127(1):21-27.

17. Mukhtar OM, Miller AP, Nanda NC, Pacifico AD, Kirklin JK, Nekkanti R. Two- and three-dimensional transesophageal echocardiographic localization of a right atrial lipoma: importance of orienting echocardiographic images to the surgeon's view. Echocardiography. 2001;18(6):539542.

18. Konen E, Merchant N, Gutierrez C, Provost Y, Mickleborough L, Paul NS, Butany J. True versus false left ventricular aneurysm: differentiation with MR imaging-initial experience. Radiology. 2005;236(1):65-70.

19. Yeo TC, Malouf JF, Reeder GS, Oh JK. Clinical characteristics and outcome in postinfarction pseudoaneurysm. Am J Cardiol. 1999;84(5):592-595, A598.

20. Zoffoli G, Mangino D, Venturini A, Terrini A, Asta A, Zanchettin C, Polesel E. Diagnosing left ventricular aneurysm from pseudo-aneurysm: a case report and a review in literature. J Cardiothorac Surg. 2009;4:11.

21. Beckerling $\mathrm{CH}$, Gibb BH, Houghton HG, Le Roux BT. Left ventricular aneurysm. Thorax. 1969;24(2):173-175.

22. Dubnow MH, Burchell HB, Titus JL. Postinfarction ventricular aneurysm. A clinicomorphologic and electrocardi- ographic study of 80 cases. Am Heart J. 1965;70(6):753760.

23. Chirillo F, Cavarzerani A, Ius P, Totis O, Bruni A, Valfre C, Stritoni P. Role of transthoracic, transesophageal, and transgastric two-dimensional and color Doppler echocardiography in the evaluation of mechanical complications of acute myocardial infarction. Am J Cardiol. 1995;76(11):833-836.

24. Dor V, Saab M, Coste P, Kornaszewska M, Montiglio F. Left ventricular aneurysm: a new surgical approach. Thorac Cardiovasc Surg. 1989;37(1):11-19.

25. Berger PB, Tuttle RH, Holmes DR, Jr., Topol EJ, Aylward $\mathrm{PE}$, Horgan JH, Califf RM. One-year survival among patients with acute myocardial infarction complicated by cardiogenic shock, and its relation to early revascularization: results from the GUSTO-I trial. Circulation. 1999;99(7):873-878.

26. Nekkanti R, Nanda NC, Zoghbi GJ, Mukhtar O, McGiffin DC. Transesophageal two- and three-dimensional echocardiographic diagnosis of combined left ventricular pseudoaneurysm and ventricular septal rupture. Echocardiography. 2002;19(4):345-349.

27. Schwengel RH, Bennett SK, Sequeira AJ, White CS, Ziskind AA. Late presentation of left ventricular pseudoaneurysm and ventricular septal defect after surgery for penetrating cardiac injury. Am Heart J. 1994;127(4 Pt 1):930-932. 